\title{
THE EMBEDDING PROBLEM FOR NOETHERIAN RINGS
}

\author{
BY LANCE W. SMALL
}

Communicated by I. N. Herstein, July 31, 1968

1. Introduction. Goldie's theorem implies that a semiprime Noetherian ring can be embedded in a semisimple Artinian ring. Furthermore, the author has shown [1] that any finitely-generated algebra over a commutative Noetherian ring can also be embedded in an Artinian ring. The question has thus arisen whether an arbitrary Noetherian ring can be so embedded. In this note we present an example to show that this is not possible. The ring constructed also fails to satisfy the descending chain condition on right annihilators.

2. Definitions and notation. In this note ring means ring with unit element, Noetherian means right Noetherian and Artinian will be right Artinian.

3. The example. We begin with a simple Noetherian domain $R$ which is not a division ring (it is well known that such exist). Let $K$ be a nonzero right ideal of $R, M=R / K$, and

$$
T(M)=\{m \in M \mid m r=0,0 \neq r \in R\} .
$$

Lemma. (1) $T(M)=M$.

(2) If $m_{1}, \cdots, m_{n} \in M$, there is a nonzero $d \in R$ such that $m_{i} d=0$, $i=1, \cdots, n$.

Proof. (1) A typical element of $M$ is of the form $a+K$. If $0 \neq k \in K$, we have by the Ore condition $c, d \in R$ such that $a c=k d$ which lies in $K$. Thus, $(a+K) c=0$.

(2) Suppose $m_{i} d_{i}=0, i=1, \cdots, n$. Again by the Ore condition $d_{1} R \cap \cdots \cap d_{n} R \neq 0$. If $0 \neq d=d_{1} r_{1}=\cdots=d_{n} r_{n}$, then it is clear that $m_{i} d=0, i=1, \cdots, n$.

Since $R$ is simple, the annihilator of $M$ is 0 . Let $\mathcal{F}=\{I \subset R \mid I$ is the annihilator of a finite subset of $M\}$.

LemMa. $\mathcal{F}$ does not satisfy the minimum condition.

Proof. If $\mathfrak{F}$ satisfied the minimum condition, there would be a minimal element $V \in \mathcal{F} . V$ is the annihilator of $\left\{m_{1}, \cdots, m_{n}\right\}$, say. If $m$ is any other element of $M$, the annihilator (which is nonzero) of $\left\{m, m_{1}, \cdots, m_{n}\right\}$ is $V \cap U$, where $U$ is the annihilator of $m$. By the choice of $V$, we have $V=V \cap U$ so that $V \subset U$ and $V$ annihilates $M$ which is a contradiction. 
We can now construct our example. Let $F$ be the center of $R$ which is, of course, a field. Define $S$ to be the ring of all two-by-two "matrices" of the form

$$
\left(\begin{array}{ll}
a & b \\
0 & c
\end{array}\right), \quad a \in F, \quad b \in M, \quad c \in R,
$$

where equality and addition are defined component-wise and the multiplication is

$$
\left(\begin{array}{ll}
a & b \\
0 & c
\end{array}\right)\left(\begin{array}{ll}
a^{\prime} & b^{\prime} \\
0 & c^{\prime}
\end{array}\right)=\left(\begin{array}{cc}
a a^{\prime} & a b^{\prime}+b c^{\prime} \\
0 & c c^{\prime}
\end{array}\right)
$$

where $R$ and $F$ act on $M$ in the obvious way.

THEOREM 1. (1) $S$ is a Noetherian ring.

(2) $S$ does not satisfy the minimum condition on right annihilators.

(3) S cannot be embedded in an Artinian ring.

Proof. (1) may be rapidly verified by the reader.

(2) If $C$ is a finite subset of $M$ and

$$
\bar{C}=\left\{\left(\begin{array}{ll}
0 & c \\
0 & 0
\end{array}\right) \mid c \in C\right\},
$$

then the right annihilator of $\bar{C}$ is

$$
\left\{\left(\begin{array}{ll}
a & b \\
0 & r
\end{array}\right) \mid a \in F, b \in M, C r=0\right\} .
$$

Thus, by the previous lemma, the right annihilators of such $\bar{C}$ do not satisfy the minimum condition.

(3) It is well known that subrings inherit the annihilator chain conditions. Hence, by (2) we are done.

Without too much labor it can be shown that $S$ cannot be embedded in any Artinian ring (right or left, with or without a unit element).

\section{REFERENCE}

1. L. W. Small, Orders in Artinian rings, J. Algebra 4 (1966), 13-41.

University of California, Berkeley, California 94720 